TITLE:

\title{
Measurement of transient photoabsorption and photocurrent of BiFeO_3 thin films: Evidence for long-lived trapped photocarriers
}

\section{$\operatorname{AUTHOR}(\mathrm{S}):$}

Yamada, Yasuhiro; Nakamura, Toru; Yasui, Shintaro; Funakubo, Hiroshi; Kanemitsu, Yoshihiko

\section{CITATION:}

Yamada, Yasuhiro ...[et al]. Measurement of transient photoabsorption and photocurrent of BiFeO_3 thin films: Evidence for long-lived trapped photocarriers. Physical Review B 2014, 89(3): 035133.

\section{ISSUE DATE:}

2014-01-21

URL:

http://hdl.handle.net/2433/180321

RIGHT:

(C)2014 American Physical Society 


\title{
Measurement of transient photoabsorption and photocurrent of $\mathrm{BiFeO}_{3}$ thin films: Evidence for long-lived trapped photocarriers
}

\author{
Yasuhiro Yamada, ${ }^{1}$ Toru Nakamura, ${ }^{1}$ Shintaro Yasui, ${ }^{2}$ Hiroshi Funakubo, ${ }^{2}$ and Yoshihiko Kanemitsu ${ }^{1, *}$ \\ ${ }^{1}$ Institute for Chemical Research, Kyoto University, Uji, Kyoto 611-0011, Japan \\ ${ }^{2}$ Interdisciplinary Graduate School of Science and Engineering, Tokyo Institute of Technology, Midori-ku, Yokohama, 226-8502, Japan
}

(Received 29 October 2013; revised manuscript received 2 January 2014; published 21 January 2014)

\begin{abstract}
We have studied the optical response and dynamical behavior of photocarriers in $\mathrm{BiFeO}_{3}$ thin films by means of transient absorption (TA) and photocurrent (PC) measurements. PC and absorption spectroscopy indicate that $\mathrm{BiFeO}_{3}$ thin films have an indirect band gap energy of $\sim 2.4 \mathrm{eV}$. The TA and PC decay dynamics have fast $(\sim 1 \mathrm{~ns})$ and slow $(\sim 100 \mathrm{~ns})$ components that are attributed to the localization of free carriers to shallow trap states and the recombination of trapped carriers, respectively. The long decay time of the PC is caused by the thermal activation of trapped carriers into the conduction band. Long-lived trapped photocarriers can be linked to the ferroelectricity and give rise to unique photoinduced phenomena in $\mathrm{BiFeO}_{3}$.
\end{abstract}

DOI: 10.1103/PhysRevB.89.035133

\section{INTRODUCTION}

Transition-metal oxides have fascinating multifunctional properties that can be employed for a range of new device concepts, and the large remnant polarization, high Curie temperature, and strong multiferroic coupling of $\mathrm{BiFeO}_{3}$ makes this oxide one of the most promising device materials [1-4]. Bulk $\mathrm{BiFeO}_{3}$ crystals take a rhombohedrally distorted perovskite structure at room temperature, and the quasitetragonal phase in $\mathrm{BiFeO}_{3}$ thin films, which are usually deposited on $\mathrm{SrTiO}_{3}$ or $\mathrm{DyScO}_{3}$ substrates, is stabilized by the compressive strain induced by the lattice mismatch with the substrate [5]. Extensive studies of the dielectric properties of $\mathrm{BiFeO}_{3}$ and related heterostructures have been performed [6,7], and it has been reported that the tetragonal phase of $\mathrm{BiFeO}_{3}$ results in an enhancement of the spontaneous polarization $[8,9]$.

The unique optical properties of $\mathrm{BiFeO}_{3}$ have also recently come under scrutiny; bulk $\mathrm{BiFeO}_{3}$ crystals exhibit a large photoinduced size expansion along the polarization direction [10,11], and a nonthermal, ultrafast piezoelectric response induced by above-band-gap photoexcitation has also been observed in time-resolved x-ray diffraction measurements [12]. These results imply that the photoexcited carriers (photocarriers) are strongly linked to the ferroelectric properties of $\mathrm{BiFeO}_{3}$. In addition, it has been reported that $\mathrm{BiFeO}_{3}$ thin films on $\mathrm{DyScO}_{3}$ display an above-band-gap photovoltage that is generated along the net polarization direction $[13,14]$. Photocarriers would thus appear to be driven by the ferroelectric depolarization field, which is the origin of the large photovoltaic effect. It has also been suggested that the photovoltaic properties of $\mathrm{BiFeO}_{3}$ are completely different from those of conventional photoconversion systems based on the built-in field of pn and Schottky junctions. The unique photoinduced phenomena observed in $\mathrm{BiFeO}_{3}$ may suggest new directions for ferroelectrics science and assist in the development of innovative ferroelectrics-based devices, including light-controlled elastic actuators and lightenergy converters. However, the fundamental optical properties and photocarrier recombination processes of $\mathrm{BiFeO}_{3}$

*Corresponding author: kanemitu@ @scl.kyoto-u.ac.jp
PACS number(s): 78.47.jb, 77.55.fp, 78.47.db, 78.66.-w that determine the exact optical response are still unclear. A study of the dynamical behavior of the photocarriers is thus essential for understanding the unique photoinduced effects in $\mathrm{BiFeO}_{3}$.

In this work, we report on the relaxation and recombination dynamics of photocarriers of $\mathrm{BiFeO}_{3}$ thin films investigated using a combination of transient absorption (TA) and timeresolved photocurrent (PC) measurements. The band gap energy was determined on the basis of the PC excitation spectrum to be approximately $2.4 \mathrm{eV}$, and the TA spectrum has a strong photoabsorption peak at around $2.3 \mathrm{eV}$, which we attribute to the optical transition of photoexcited electrons to the higher conduction band. The TA dynamics show two exponential decay components under weak photoexcitation, and we assign the fast component to the localization of free photocarriers and the slow decay component to the relaxation of photocarriers in the shallow trap states. These results are consistent with both the very fast decay of the PC signals, which mainly reflect the population of free photocarriers, and the thermal activation of the trapped carriers that gives rise to a long PC lifetime. Here, we discuss the photocarrier relaxation and recombination processes and their impact on the photoinduced phenomena in $\mathrm{BiFeO}_{3}$.

\section{SAMPLE AND EXPERIMENTAL SETUP}

$\mathrm{BiFeO}_{3}$ epitaxial thin films were fabricated by chemical vapor deposition [15]. Because the structural and ferroelectric properties of $\mathrm{BiFeO}_{3}$ thin films are dependent on the quality and crystal structure of the substrate, we fabricated thin films on two different substrates $\left(\mathrm{SrTiO}_{3}\right.$ and $\left.\mathrm{DyScO}_{3}\right)$. The film thicknesses were $260 \mathrm{~nm}$ and $50 \mathrm{~nm}$ for the $\mathrm{SrTiO}_{3}$ and $\mathrm{DyScO}_{3}$-substrate samples, respectively. We measured the TA dynamics using femtosecond white-light pump-probe spectroscopy; pump and probe pulses with a delay time $\Delta t$ were focused onto the sample surface, and the variation in the probe-pulse intensity induced by the pump excitation was detected as a function of $\Delta t$. The white-light probe pulses were generated by focusing a 1.2-eV laser pulse onto a sapphire plate. The pump energy was fixed at $3.1 \mathrm{eV}$. A wavelengthtunable femtosecond laser system based on a $\mathrm{Yb}: \mathrm{KGW}$ 
(potassium gadolinium tungstate) regenerative amplified laser with a pulse duration of $300 \mathrm{fs}$ was used as the light source. Transient absorption measurements in the microsecond region were also performed using a 2.3-eV continuous wave (cw) laser as the probe beam. For the PC measurements, two parallel rectangular $\mathrm{Al}$ electrodes were evaporated onto the sample surface, and femtosecond laser pulses $(3.1 \mathrm{eV})$ were focused onto the gap between the electrodes [16]. The transient PC was recorded by an oscilloscope and a current-voltage converter. The time resolution of the PC measurement was about 6 ns.

\section{RESULTS AND DISCUSSION}

The band gap energy of $\mathrm{BiFeO}_{3}$ has previously been reported to lie within a range of 2.4 to $2.8 \mathrm{eV}$, but optical absorption in the shallow trap states formed by defects and impurities smears the optical absorption edge that corresponds to the band gap energy, which makes it difficult to determine the exact band gap energy [17-20]. It has also been pointed out that the optical absorption peak due to a $d-d$ transition appears in the near band-edge absorption spectrum of $\mathrm{BiFeO}_{3}[19,20]$. The optical absorption and $\mathrm{PC}$ spectra of $\mathrm{BiFeO}_{3}$ thin films on the $\mathrm{SrTiO}_{3}$ (BFO/STO) and $\mathrm{DyScO}_{3}$ (BFO/DSO) substrates are shown in Fig. 1. The optical absorption spectrum of $\mathrm{BFO} / \mathrm{STO}$ shows a significant increase above $2.4 \mathrm{eV}$, which is
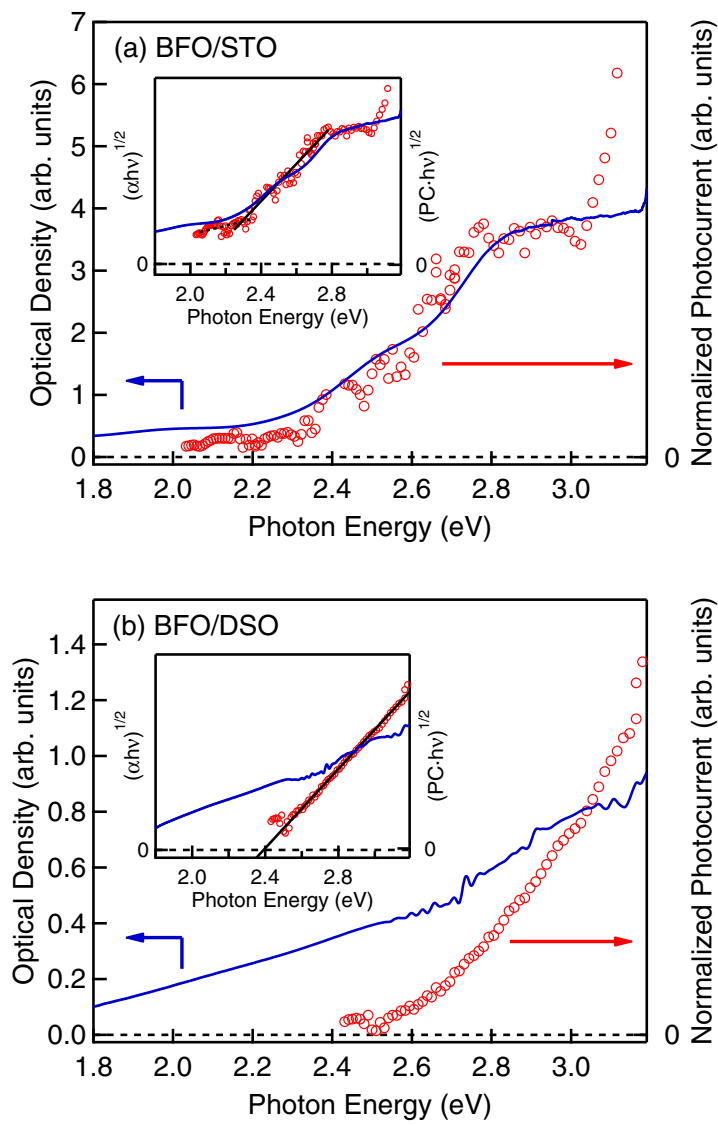

FIG. 1. (Color online) Optical absorption and PC excitation spectra of (a) $\mathrm{BiFeO}_{3} / \mathrm{SrTiO}_{3}$ and (b) $\mathrm{BiFeO}_{3} / \mathrm{DyScO}_{3}$. The insets show Tauc plots of the optical absorption and PC. The dashed lines are a guide for the eye. consistent with reported values of the band gap energy [20,21] and also has a long tail on the low-energy side. The BFO/DSO, however, shows no clear absorption edge around $2.4 \mathrm{eV}$, and the optical absorption below the band gap energy suggests that there is a high density of below band gap states introduced by defects due to the lattice mismatch. The onsets of the PC spectra (Fig. 1) of BFO/STO and BFO/DSO are approximately 2.3 and $2.4 \mathrm{eV}$, respectively. As the $\mathrm{PC}$ reflects the optical absorption that contributes to the PC generated by band-toband photoexcitation, it is therefore less affected by the optical absorption of deep defect or impurity states. In other words, the $\mathrm{PC}$ is more sensitive to band-edge optical transitions. Tauc plots of the PC (insets in Fig. 1) reveal a linear variation in the $\mathrm{PC}$ of both $\mathrm{BFO} / \mathrm{STO}$ and $\mathrm{BFO} / \mathrm{DSO}$, which is evidence of an indirect transition [22,23]. This assignment is consistent with our experimental results that show that almost no photoluminescence is observed from $\mathrm{BiFeO}_{3}$ thin films even at low temperatures (data not shown) because most direct-gap semiconductors exhibit efficient light emission. The indirect gap and the band gap energy are also consistent with theoretical band calculations [21,24]. In an indirect-gap semiconductor, optical absorption involves wavenumber-conserving phonons, and we estimate $\left(E_{g}-E_{p h}\right)$ to be $2.4 \mathrm{eV}$, where $E_{g}$ and $E_{p h}$ are the band gap energy and the phonon energy, respectively. As the phonon energy is typically much smaller than the band gap energy, we conclude that the band gap energy of the $\mathrm{BiFeO}_{3}$ thin film is approximately $2.4 \mathrm{eV}$ at $300 \mathrm{~K}$.

Note that the large difference in the optical absorption and PC spectra of BFO/STO and BFO/DSO arises from the differences in the quality of the substrate rather than interface strain due to the lattice mismatch. The below-bandedge optical properties are generally sensitive to defects and impurities; therefore, we consider the defect (impurity) density to be strongly dependent on the substrate. However, the band gap energy, which is dependent on the crystal structure, is relatively unaffected by the substrate. This result is consistent with the results of a previous report [25].

Femtosecond TA measurements were performed with an excitation photon energy $(3.1 \mathrm{eV})$ that was higher than the band gap energy, and Fig. 2 shows the change in the optical density $(\triangle \mathrm{OD})$ spectra of BFO/STO and BFO/DSO for different delay times. In both samples, we observed a photoabsorption peak at around $2.3 \mathrm{eV}$. The absence of photobleaching, which generally appears near the band gap energy of direct-gap semiconductors under strong photoexcitation conditions, is consistent with our assignment that $\mathrm{BiFeO}_{3}$ is an indirect-gap semiconductor. The $2.3-\mathrm{eV}$ photoabsorption peak appears just after photoexcitation, suggesting that photocarriers in the band state (not localized carriers) are responsible for the absorption because there are no localized carriers just after the excitation. We consider that the $2.3-\mathrm{eV}$ peak corresponds to the optical transition of electrons in the lowest conduction band to higher energy levels; a higher band composed mainly of the Bi $6 p$ state has been theoretically predicted [26]. With an increase in the delay time, the $2.3-\mathrm{eV}$ peak shows a blueshift owing to the crossover of two TA bands. For BFO/STO, these two TA bands peak at 2.27 and $2.41 \mathrm{eV}$ [as shown by the dashed curves in Fig. 2(a)], and we attribute these peaks to optical transitions from the near band-edge (shallow) state and the lowest energy conduction band to a higher conduction band, 

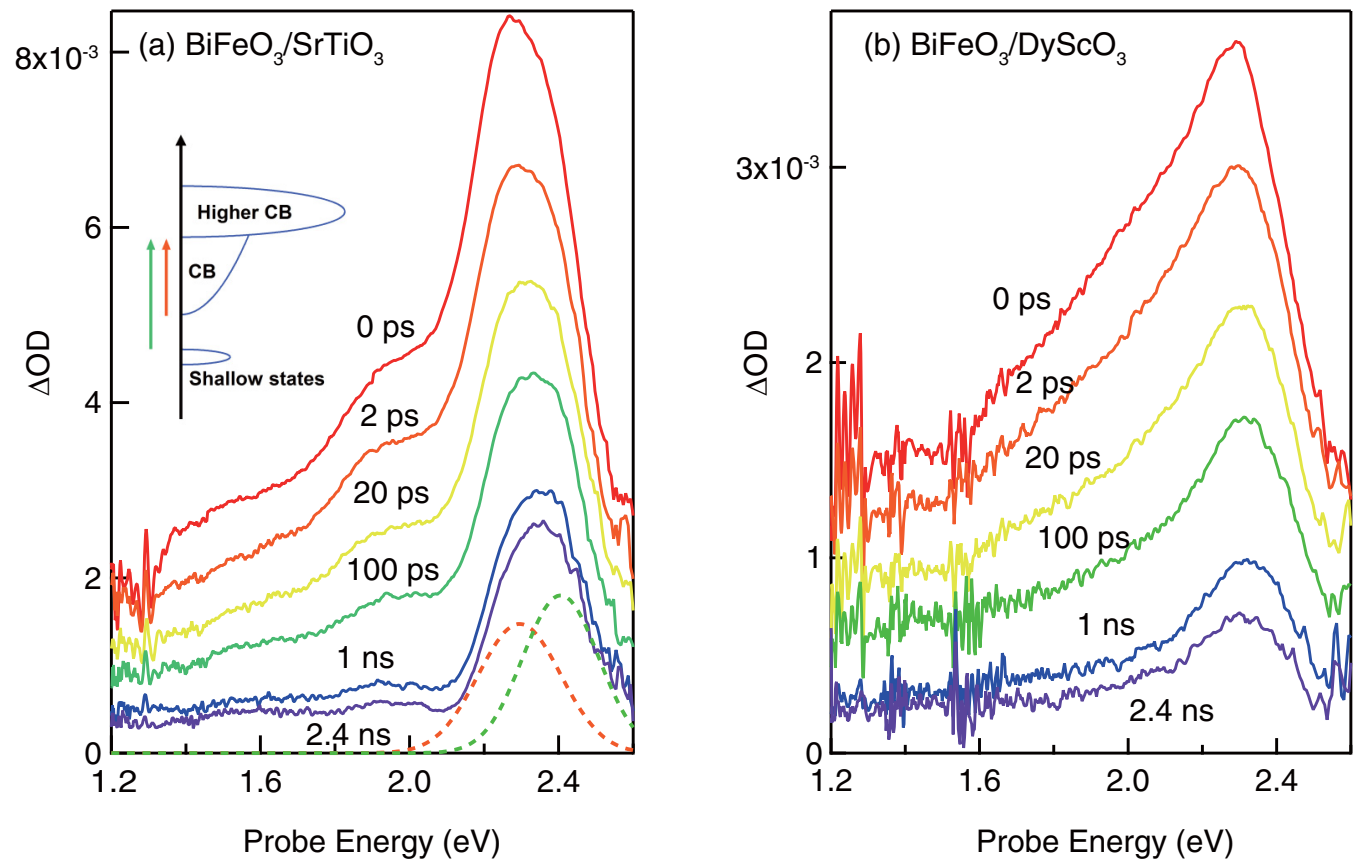

FIG. 2. (Color online) $\mathrm{TA}$ spectra of (a) $\mathrm{BiFeO}_{3} / \mathrm{SrTiO}_{3}$ and (b) $\mathrm{BiFeO}_{3} / \mathrm{DyScO}_{3}$ for different delay times. Dashed curves represent the two Gaussian functions that compose the 2.3-eV TA band. The inset illustrates the energy levels of $\mathrm{BiFeO}_{3}$.

respectively, as indicated by the arrows in the inset. The TA band crossover indicates that photocarriers in the band state relax to the shallow trap state.

The TA dynamics are shown in Fig. 3 for a probe energy of $2.3 \mathrm{eV}$ (photoabsorption peak energy) and different excitation densities; the TA intensities were normalized at $200 \mathrm{ps}$. The TA decay profiles in the nanosecond time range, obtained from the cw-probe TA measurements, are shown in the inset. The TA intensity at $2.3 \mathrm{eV}$ corresponds to the carrier density at the band state and shallow trap state. Between 0 and 200 ps, the TA decay profiles are dependent on the excitation density; a very fast decay component appears with an increase in the excitation density. This excitation-density-dependent decay component can be associated with Auger recombination involving three carriers under strong photoexcitation conditions [27,28].

Under weak photoexcitation fluence, the TA dynamics consist of fast and slow components. The lifetime of the fast component is about $1 \mathrm{~ns}$ in both samples, as determined by a single-exponential fitting over the range 0.2-2 ns. However, the slow component has a nonexponential decay profile. We obtained average slow lifetimes of $130 \mathrm{~ns}$ for BFO/STO and 160 ns for BFO/DSO by fitting a double exponential over the range 10-800 ns. Additional decay components with a longer decay time also exist, and these can be attributed to relaxation in the deeper states. Note that these very slow decay components are sensitive to the probe energy and focal position of the laser. We attribute the fast and slow decay components to the localization of free photocarriers and the relaxation of photocarriers in the shallow trap, respectively, as shown schematically in Fig. 4(a); according to our model, photoexcited electrons rapidly relax into shallow trap states $(\sim 1 \mathrm{~ns})$ and reach thermal equilibrium, which is consistent with the nanosecond timescale of the TA crossover. These trapped carriers have a longer lifetime $(\sim 130-160 \mathrm{~ns})$, and thermal activation of carriers in the shallow trap induces transitions to the conduction band that are reflected by the slow decay component.

To confirm our photocarrier recombination model described in Fig. 4(a), we examined the PC decay profiles of BFO/STO under different bias voltages [Fig. 4(b)]. It should be noted that we were careful in focusing the excitation laser onto the gap between the electrodes to avoid illuminating and heating the electrodes, which would induce a pyroelectric current independent of the bias voltage. The observed PC was very weak because of the large gap width needed to reduce the pyroelectric current. The PC increases linearly with the bias voltage, meaning that an ohmic contact is formed at the interface of the $\mathrm{BiFeO}_{3}$ sample and electrodes. The PC decay profiles are almost independent of the bias voltage. Two decay components can be observed in the PC profiles: a very fast decay component that decays within $\sim 6$ ns (equivalent to the time resolution of the measurements) and a slow decay component with an estimated decay time of $200 \mathrm{~ns}$ (determined from a single-exponential fitting). The BFO/DSO sample showed similar PC decay dynamics, and the decay times were similar to those estimated from the TA results.

The logarithm of the time-integrated PC intensity as a function of inverse temperature [Fig. 4(b) inset] reveals a linear relationship that indicates the existence of a thermally activated process from the shallow trap state to the conduction band, which supports the recombination model proposed in Fig. 4(a). We found that the activation energies $\left(E_{a}\right)$ of BFO/STO and $\mathrm{BFO} / \mathrm{DSO}$ were 120 and $80 \mathrm{meV}$, respectively, based on an Arrhenius plot of the PC intensity; an activation energy of about $100 \mathrm{meV}$ corresponds to the energy difference between the band state and shallow trap state, as well as being consistent with the energy shift of the photoabsorption peak (around 

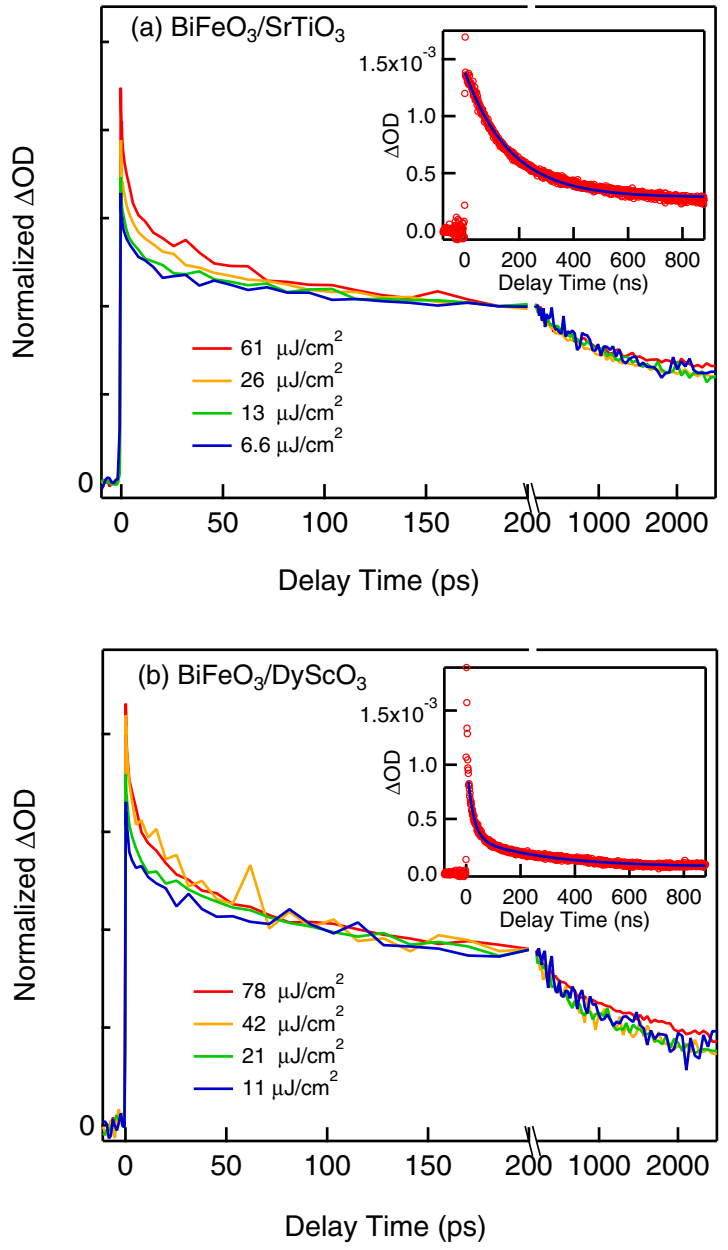

FIG. 3. (Color online) Normalized TA decay profiles of (a) $\mathrm{BiFeO}_{3} / \mathrm{SrTiO}_{3}$ and (b) $\mathrm{BiFeO}_{3} / \mathrm{DyScO}_{3}$ for different excitation densities. The inset shows the TA decay profiles in the submicrosecond time region and the calculated double-exponential function (blue curve).

(a)

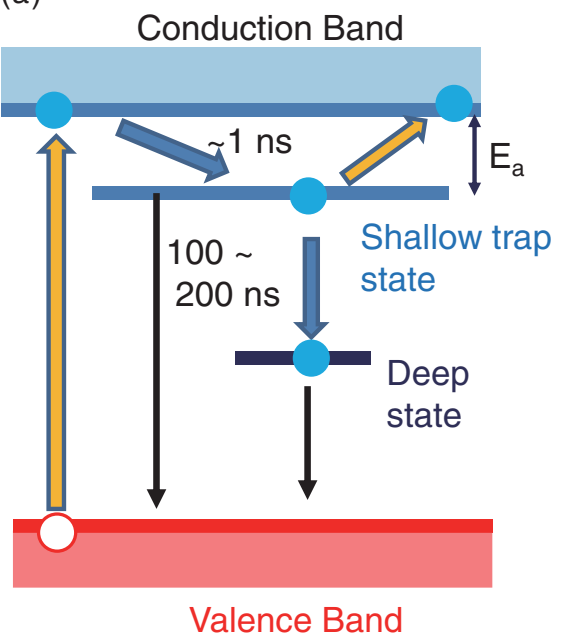

$100 \mathrm{meV}$ ) shown in Fig. 2. These results provide further support for our photocarrier recombination model. Note that the substrate-dependent activation energy can be attributed to the below band gap state, which is also dependent on the substrate, as shown in the PC and absorption spectra (see Fig. 1).

Our results indicate that the free photocarriers are captured in the shallow trap state within 1 ns. However, at room temperature, carriers in the shallow trap state have a long lifetime (130 and $160 \mathrm{~ns}$ for BFO/STO and BFO/DSO, respectively), and thermally activated free carriers have an effectively longer lifetime. Owing to these long-lived carriers, a high carrier density is achieved when the carriers are continuously photogenerated. This long carrier lifetime is linked to the ferroelectricity of $\mathrm{BiFeO}_{3}$; photogenerated electrons and holes will be spatially separated due to the depolarized field in ferroelectric $\mathrm{BiFeO}_{3}$, which reduces the photocarrier recombination rates, and more effective charge separation would be expected at ferroelectric domain walls because of the large associated electric field. Our research suggests that extremely long-lived carriers in the trap states play an important role in the photoinduced phenomena such as the size expansion and large photovoltage mentioned in Refs. 10-14. Photocarriers driven by ferroelectricity would appear to be responsible for the unique photoresponses in ferroelectric semiconductors.

\section{CONCLUSION}

We have studied the near-band-edge optical properties and dynamical behavior of photocarriers in $\mathrm{BiFeO}_{3}$ thin films on $\mathrm{SrTiO}_{3}$ and $\mathrm{DyScO}_{3}$ substrates by means of transient TA and PC measurements. We did not observe any significant difference in the optical responses of the two $\mathrm{BiFeO}_{3}$ thin films, and we found that the TA decay profiles have two components. The fast and slow decay components were attributed to the relaxation of free photocarriers and the recombination of photocarriers in the shallow traps, respectively. These results are consistent with the PC decay dynamics, which are more sensitive to the mobile carrier population. The fast localization

(b)

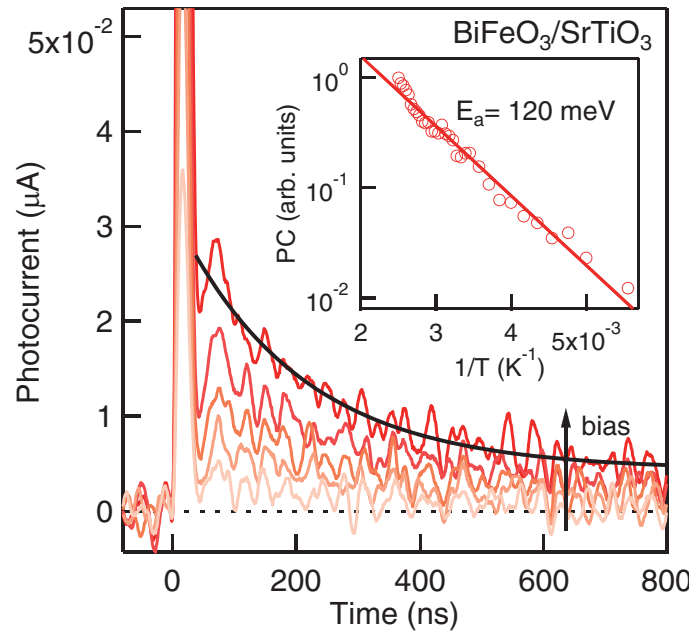

FIG. 4. (Color online) (a) Schematic of the photocarrier relaxation and recombination processes in $\mathrm{BiFeO}_{3}$. (b) $\mathrm{PC}$ dynamics of $\mathrm{BiFeO}_{3} / \mathrm{SrTiO}_{3}$ at different bias voltages $(2,4,6,8$, and $10 \mathrm{~V})$. The inset shows the time-integrated $\mathrm{PC}$ as a function of inverse temperature. 
of free photocarriers is related to the very small PC observed in the photovoltaic measurements. While free photocarriers are rapidly trapped within $1 \mathrm{~ns}$, trapped carriers are thermally activated and have an effectively long lifetime. We believe that our results provide a deep insight into the photoinduced phenomena in $\mathrm{BiFeO}_{3}$.

\section{ACKNOWLEDGMENTS}

Part of this work was supported by The Sumitomo Electric Industries Group CSR Foundation, KAKENHI (No. 24740202 and No. 25247052), the Collaborative Research Program of Institute for Chemical Research, Kyoto University (No. 2011-6 and No. 2012-7), and JST-CREST.
[1] As a review, G. Catalan and J. F. Scott, Adv. Mater. 21, 2463 (2009).

[2] D. Lebeugle, D. Colson, A. Forget, and M. Viret, Appl. Phys. Lett. 91, 022907 (2007).

[3] Y.-H. Chu, L. W. Martin, M. B. Holcomb, M. Gajek, S.-J. Han, Q. He, N. Balke, C.-H. Yang, D. Lee, W. Hu, Q.Zhan, P.-L. Yang, A. Fraile-Rodríguez, A. Scholl, S. X. Wang, and R. Ramesh, Nat. Mater. 7, 478 (2008).

[4] N. Balke, S. Choudhury, S. Jesse, M. Huijben, Y. H. Chu, A. P. Baddorf, L. Q. Chen, R. Ramesh, and S. V. Kalinin, Nat. Nanotechnol. 4, 868 (2009).

[5] R. J. Zeches, M. D. Rossell, J. X. Zhang, A. J. Hatt, Q. He, C.-H. Yang, A. Kumar, C. H. Wang, A. Melville, C. Adamo, G. Sheng, Y.-H. Chu, J. F. Ihlefeld, R. Erni, C. Ederer, V. Gopalan, L. Q. Chen, D. G. Schlom, N. A. Spaldin, L. W. Martin, and R. Ramesh, Science 326, 977 (2009).

[6] Y. P. Wang, L. Zhou, M. F. Zhang, X. Y. Chen, J.-M. Liu, and Z. G. Liu, Appl. Phys. Lett. 84, 1731 (2004).

[7] M. J. Calderón, S. Liang, R. Yu, J. Salafranca, S. Dong, S. Yunoki, L. Brey, A. Moreo, and E. Dagotto, Phys. Rev. B, 84, 024422 (2011).

[8] J. X. Zhang, Q. He, M. Trassin, W. Luo, D. Yi, M. D. Rossell, P. Yu, L. You, C. H. Wang, C. Y. Kuo, J. T. Heron, Z. Hu, R. J. Zeches, H. J. Lin, A. Tanaka, C. T. Chen, L. H. Tjeng, Y.-H. Chu, and R. Ramesh, Phys. Rev. Lett. 107, 147602 (2011).

[9] J. Wang, J. B. Neaton, H. Zheng, V. Nagarajan, S. B. Ogale, B. Liu, D. Viehland, V. Vaithyanathan, D. G. Schlom, U. V. Waghmare, N. A. Spaldin, K. M. Rabe, M. Wuttig, and R. Ramesh, Science 299, 1719 (2003).

[10] B. Kundys, M. Viret, D. Colson, and D. O. Kundys, Nat. Mater. 9, 803 (2010).

[11] B. Kundys, M. Viret, C. Meny, V. Da Costa, D. Colson, and B. Doudin, Phys. Rev. B 85, 092301 (2012).

[12] H. Wen, P. Chen, M. P. Cosgriff, D. A. Walko, J. H. Lee, C. Adamo, R. D. Schaller, J. F. Ihlefeld, E. M. Dufresne, D. G. Schlom, P. G. Evans, J. W. Freeland, and Y. Li, Phys. Rev. Lett. 110, 037601 (2013).
[13] S. Y. Yang, J. Seidel, S. J. Byrnes, P. Shafer, C.-H. Yang, M. D. Rossell, P. Yu, Y.-H. Chu, J. F. Scott, J. W. Ager, III, L. W. Martin, and R. Ramesh, Nat. Nanotechnol. 5, 143 (2010).

[14] J. Seidel, D. Fu, S.-Y. Yang, E. Alarcón-Lladó, J. Wu, R. Ramesh, and J. W. Ager, III, Phys. Rev. Lett. 107, 126805 (2011).

[15] R. Ueno, S. Okamura, H. Funakubo, and K. Saito, Jpn. J. Appl. Phys. 44, L1231 (2005).

[16] Y. Yamada and Y. Kanemitsu, Appl. Phys. Lett. 101, 133907 (2012).

[17] S. J. Clark and J. Robertson, Appl. Phys. Lett. 90, 132903 (2007).

[18] L. Bi, A. R. Taussig, H.-S. Kim, L. Wang, G. F. Dionne, D. Bono, K. Persson, G. Ceder, and C. A. Ross, Phys. Rev. B 78, 104106 (2008).

[19] P. Chen, N. J. Podraza, X. S. Xu, A. Melville, E. Vlahos, V. Gopalan, R. Ramesh, D. G. Schlom, and J. L. Musfeldt, Appl. Phys. Lett. 96, 131907 (2010).

[20] B. Ramachandran, A. Dixit, R. Naik, G. Lawes, and M. S. Ramachandra Rao, Phys. Rev. B 82, 012102 (2010).

[21] J. B. Neaton, C. Ederer, U. V. Waghmare, N. A. Spaldin, and K. M. Rabe, Phys. Rev. B 71, 014113 (2005).

[22] P. Y. Yu and M. Cardona, Fundamentals of Semiconductors, 3rd ed. (Springer, Berlin, 2005).

[23] Y. Yamada and Y. Kanemitsu, Phys. Rev. B 82, 121103(R) (2010).

[24] R. V. Pisarev, A. S. Moskvin, A. M. Kalashnikova, and T. Rasing, Phys. Rev. B 79, 235128 (2009).

[25] H. L. Liu, M. K. Lin, Y. R. Cai, C. K. Tung, and Y. H. Chu, Appl. Phys. Lett. 103, 181907 (2013).

[26] O. Diéguez, O. E. González-Vázquez, J. C. Wojdeł, and J. Íñiguez, Phys. Rev. B 83, 094105 (2011).

[27] P. T. Landsberg, Recombination in Semiconductors (Cambridge University Press, Cambridge, 1991).

[28] Y. Yamada, H. Yasuda, T. Tayagaki, and Y. Kanemitsu, Phys. Rev. Lett. 102, 247401 (2009). 